\title{
Some Examples of Projective and c-projective Compactifications of Einstein Metrics
}

\author{
Maciej Dunajski, A. Rod Gover and Alice Waterhouse
}

\begin{abstract}
We construct several examples of compactifications of Einstein metrics. We show that the Eguchi-Hanson instanton admits a projective compactification which is non-metric, and that a metric cone over any (pseudo)-Riemannian manifolds admits a metric projective compactification. We construct a para-c-projective compactification of neutral signature Einstein metrics canonically defined on certain rank- $n$ affine bundles $M$ over $n$-dimensional manifolds endowed with projective structures.
\end{abstract}

\section{Introduction}

There are several notions of compactifications of a (pseudo) Riemannian manifold $(M, g)$. In a conformal compactification $(\bar{M}, \bar{g})$, one has that $\bar{M}$ is a manifold with boundary such that $M$ is the interior of $\bar{M}$, and there is a defining function $T$ for the boundary such that the metric $\bar{g}=T^{2} g$ smoothly extends to the boundary $\partial M$ of $\bar{M}$ (T being a defining function for $\partial M$ means that $\partial M=\mathcal{Z}(T):=\{p \in \bar{M}: T(p)=0\}$ and $\mathrm{d} T$ is nowhere zero on $\partial M$.) The geodesics of $g$ do not correspond to geodesics of $\bar{g}$, but the angles are preserved. This kind of compactification has proven to be useful in studying the causal structure of space times in general relativity [21], and quantum field theory [23]. It also underlies formulating the boundary conditions [22] of conformally invariant field equations like the Yang-Mills theory where the curvature decay rate on $\mathbb{R}^{4}$ is equivalent to the connection extending to a one-point compactification $\overline{\mathbb{R}}^{4}=\mathbb{R}^{4} \cup\{\infty\}=S^{4}$. In this case, the round metric on $S^{4}$ is conformally equivalent to a flat Euclidean metric on $\mathbb{R}^{4}$.

The conformal compactification is not available or not natural for many complete metrics that one may want to compactify, which motivates a search 
for other compactification mechanisms. In a projective compactification of a pseudo-Riemannian manifold [6], the unparametrised geodesics of $(M, g)$ smoothly extend to a boundary $\partial M$ of the manifold $\bar{M}=M \cup \partial M$ (see Sect. 2 for definitions). In general, this manifold does not carry a metric, but only an affine connection $\bar{\nabla}$ belonging to the projective equivalence class containing the Levi-Civita connection of $g$. This kind of compactification is naturally applicable to scattering problems [19].

There are two related concepts of compactifications: In a $c$-projective compactification [7] of an almost complex manifold $(M, J)$ with complex connection $\nabla$, the compactifying connection $\bar{\nabla}$ belongs to the $c$-projective equivalence class of $\nabla$, i.e. $\nabla$ and $\bar{\nabla}$ preserve $J$, have the same torsion, and share the same $J$-planar curves (see $\S 3$ for definitions). In the para- $c$-projective compactification which we shall introduce in Sect. 3, the endomorphism $J: T M \rightarrow T M$ squares to identity. The boundary $\partial M$ acquires a contact structure, and a conformal metric on the contact distribution.

This paper is organised as follows. In Sect. 2, we shall review the notion of the projective compactification and show that the Eguchi-Hanson gravitational instanton can be projectively compactified. We shall then introduce the notion of a metric projective compactification, where the connection $\bar{\nabla}$ is the Levi-Civita connection of some metric $\bar{g}$ on $\bar{M}$. We shall prove (Theorem 2.4) that a metric cone over a (pseudo)-Riemannian manifold admits a metric projective compactification. In Sects. 3 and 4, we shall construct a para-c-projective compactification of a neutral signature Einstein metric $g$ defined on a projectivised tractor bundle $M$ of any projective structure (Theorems 4.2 and 4.3). The model for this construction will be the compactification of $M=S L(n+1) / G L(n)$ which corresponds to the flat projective structure on $\mathbb{R} \mathbb{P}^{n}$.

\section{Metric and non-metric projective compactifications}

In this section, we shall introduce the concept of a metric projective compactification of a manifold $M$ with an affine connection $\nabla$ and give some examples of metric and non-metric projective compactifications.

Definition 2.1. An affine connection $\nabla$ on $M$ admits a projective compactification of order $\alpha$ to a manifold with boundary $\bar{M}=M \cup \partial M$ if there exists a function $T: \bar{M} \rightarrow \mathbb{R}$ such that $T=0$ is the boundary $\partial M \subset \bar{M}$, the differential $\mathrm{d} T$ does not vanish on $\partial M$, and a projectively equivalent connection $\bar{\nabla}$ on $M$ defined by

$$
\bar{\nabla}_{X} Y=\nabla_{X} Y+\Upsilon(X) Y+\Upsilon(Y) X
$$

with

$$
\Upsilon=\frac{\mathrm{d} T}{\alpha T}
$$

extends smoothly to $\partial M$. 
In $[6]$, it was shown that if $\nabla$ is the Levi-Civita connection of a (pseudo)Riemannian metric $g$ on $M$, then a sufficient condition for a projective compactification of order $\alpha>0$ (such that $2 / \alpha \in \mathbb{Z}$ ) to exist is that near the boundary $\mathcal{Z}(T)$ the metric $g$ can be put in the form

$$
g=C \frac{\mathrm{d} T^{2}}{T^{4 / \alpha}}+\frac{1}{T^{2 / \alpha}} h,
$$

for some $h$ which smoothly extends to the boundary $\mathcal{Z}(T)$ and restricts to a pseudo-Riemannian metric there, and some constant $C$. Moreover if $g$ is Ricci-flat, and a projective compactification exists, then necessarily $\alpha=1$.

In the examples below, we shall make use of a stronger notion of metric projective compactifications

Definition 2.2. A projective compactification from Definition 2.1 is metric if $\bar{\nabla}$ is the Levi-Civita connection of some (pseudo)-Riemannian metric $\bar{g}$ on $\bar{M}$.

\subsection{Example: Flat Space}

Consider a flat metric on $\mathbb{R}^{n}$ of the form

$$
g_{\text {flat }}=\mathrm{d} r^{2}+r^{2} \gamma_{S^{n-1}}
$$

where $\gamma_{S^{n-1}}$ is the round metric on a sphere $S^{n-1}$ with Ricci scalar equal to $(n-1)(n-2)$. Setting $T=r^{-1}$ puts $g_{\text {flat }}$ in the form $(2.2)$, but the resulting connection (2.1) is not metric ${ }^{1}$. To construct a metric projectivecompactification consider a defining function given by

$$
T=\frac{1}{\sqrt{r^{2}+1}}
$$

The metric $g_{\text {flat }}$ takes the form

$$
g_{\text {flat }}=\frac{\mathrm{d} T^{2}}{T^{4}}+\frac{1}{T^{2}}\left(\left(1-T^{2}\right) \gamma_{S^{n-1}}+\frac{1}{1-T^{2}} \mathrm{~d} T^{2}\right)
$$

and $h$ reduces to $\gamma_{S^{n-1}}$ on the boundary $\mathcal{Z}(T)$. It can now be verified by direct calculation that the connection (2.1) with $\Upsilon=T^{-1} \mathrm{~d} T$ is the LeviCivita connection of the metric

$$
\bar{g}=\frac{\mathrm{d} r^{2}}{\left(1+r^{2}\right)^{2}}+\frac{r^{2}}{r^{2}+1} \gamma_{S^{n-1}}=\frac{\mathrm{d} T^{2}}{1-T^{2}}+\left(1-T^{2}\right) \gamma_{S^{n-1}} .
$$

The metric $\bar{g}$ has constant positive curvature, is defined on an open set of a round sphere $S^{n}$, and extends to the boundary $T=0$ where it induces the metric $\gamma_{S^{n-1}}$.

This construction has the following natural geometric interpretation (Figure 2.1). Consider a central projection $\pi$ from a hemisphere $\mathcal{S} \subset S^{n}$ to $\mathbb{R}^{n}$. If the metric on $S^{n}$ is

$$
g_{S^{n}}=\mathrm{d} \theta^{2}+\cos \theta^{2} \gamma_{S^{n-1}}
$$

\footnotetext{
${ }^{1}$ To show this, compute the Ricci tensor $\bar{R}$ of (2.1). If (2.1) was metric for some metric $\bar{g}$ then $\bar{R}$ would be a Ricci tensor of $\bar{g}$, and so (as $\bar{g}$ is projectively flat) it would have to be a constant multiple of $\bar{g}$ by the Beltrami theorem. Computing the Ricci tensor of the metric given by $\bar{R}$ shows that it is impossible for any $\alpha$.
} 


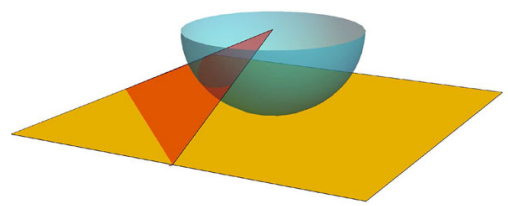

Figure 1. Central projection

and the inverse map $\pi^{-1}: \mathbb{R}^{n} \rightarrow \mathcal{S}$ is given by $\cos ^{2} \theta=r^{2}\left(r^{2}+1\right)^{-1}$ then the pull back of $\left(\pi^{-1}\right)^{*} g_{S^{n+1}}$ to $\mathbb{R}^{n+1}$ is given by $(2.4)$.

\subsection{Example: The Eguchi-Hanson Manifold}

The Eguchi-Hanson metric [14] is given by ${ }^{2}$

$$
g=\left(1-\frac{a^{4}}{r^{4}}\right)^{-1} \mathrm{~d} r^{2}+\frac{1}{4} r^{2}\left(1-\frac{a^{4}}{r^{4}}\right) \sigma_{3}^{2}+\frac{1}{4} r^{2}\left(\sigma_{1}^{2}+\sigma_{2}^{2}\right) .
$$

The apparent singularity at $r=a$ is removed by allowing

$$
r>a, \quad 0 \leq \psi \leq 2 \pi, \quad 0 \leq \phi \leq 2 \pi, \quad 0 \leq \theta \leq \pi .
$$

Setting $\rho^{2}=r^{2}\left(1-(a / r)^{4}\right)$ and expanding the metric near $r=a$ and fixing $(\theta, \phi)$ gives $g \sim\left(d \rho^{2}+\rho^{2} d \psi^{2}\right) / 4$. In the standard spherical polar coordinates, $\psi$ has a period $4 \pi$ on $S U(2)$. In our case, the period of $\psi$ is $2 \pi$ to achieve regularity. Therefore, the surfaces of constant $r$ are real projective planes defined by identifying the antipodal points on the sphere, $\mathbb{R P}^{3}=S^{3} / \mathbb{Z}^{2}$. At large values of $r$, the metric looks like $\mathbb{R}^{4} / \mathbb{Z}^{2}$ rather than Euclidean space. Thus the Eguchi-Hanson metric is an example of the asymptotically locally Euclidean manifold (see e.g. [1] for a discussion of this class of manifolds in the context of twistor theory).

To projectively compactify (2.6) and introduce the defining function

$$
T=\frac{1}{r}
$$

so that (2.6) takes the form (2.2) with

$$
h=\frac{4 a^{4} T^{6}}{1-a^{4} T^{4}} \mathrm{~d} T^{2}+\left(1-a^{4} T^{4}\right) \sigma_{3}^{2}+\sigma_{1}^{2}+\sigma_{2}^{2} .
$$

and the topology of the boundary is $\mathbb{R P}^{3}$. The resulting connection (2.1) is non-metric. One can ask whether there exists another choice of the defining function which leads to a metric projective compactification. The answer to

\footnotetext{
${ }^{2}$ Left invariant one-forms $\sigma_{i}, i=1,2,3$ on the group manifold $S U(2)$ satisfy

$$
d \sigma_{1}+\sigma_{2} \wedge \sigma_{3}=0, \quad d \sigma_{2}+\sigma_{3} \wedge \sigma_{1}=0, \quad d \sigma_{3}+\sigma_{1} \wedge \sigma_{2}=0 .
$$
}

These one-forms can be represented in terms of Euler angles by

$$
\sigma_{1}+i \sigma_{2}=e^{-i \psi}(\mathrm{d} \theta+i \sin \theta \mathrm{d} \phi), \quad \sigma_{3}=d \psi+\cos \theta \mathrm{d} \phi,
$$

where to cover $S U(2)=S^{3}$ we require the ranges

$$
0 \leq \theta \leq \pi, \quad 0 \leq \phi \leq 2 \pi, \quad 0 \leq \psi \leq 4 \pi .
$$


that question is negative, as Eguchi-Hanson is geodesically rigid: up to a constant non-zero multiple there exists only one metric in its projective class. This follows from a combination of the following two facts: (A) Two Ricciflat metrics in dimension four are projectively equivalent iff they are affinely equivalent (i.e. they share the same Levi-Civita connection). (B) In the positive signature, two affine equivalent Ricci-flat metrics are flat. See [18] for proofs of these facts.

\subsection{Metric Cones}

The projective equivalence of (2.4) and (2.3) is an example of the following result of Levi-Civita [17].

Proposition 2.3. The metrics

$$
g=\mathrm{d} r^{2}+f(r) \gamma, \quad \text { and } \quad \bar{g}=\frac{1}{(\kappa f(r)+1)^{2}} \mathrm{~d} r^{2}+\frac{f(r)}{\kappa f(r)+1} \gamma
$$

are projectively equivalent for any constant $\kappa$. Here $f$ is an arbitrary function of $r$, and $\gamma$ is an arbitrary $r$-independent metric.

Proof. One way to establish this proposition is to observe that Levi-Civita connections of $g$ and $\bar{g}$ are related by (2.1) with

$$
\Upsilon=-\frac{\kappa}{2(1+\kappa f(r))} \frac{d f}{\mathrm{~d} r} \mathrm{~d} r
$$

Let $(N, \gamma)$ be a (pseudo) Riemannian manifold of dimension $(n-1)$. A metric cone of $(N, \gamma)$ is a (pseudo) Riemannian manifold $M=N \times \mathbb{R}^{+}$with the metric

$$
g=\mathrm{d} r^{2}+r^{2} \gamma
$$

We shall use Proposition 2.3 to prove the following

Theorem 2.4. The metric cone $(M, g)$ given by (2.7) of a (pseudo)-Riemannian manifold $(N, \gamma)$ admits a metric projective compactification of order 1 .

Proof. Taking the defining function to be

$$
T=\frac{1}{\sqrt{r^{2}+1}}
$$

leads to

$$
g=\frac{\mathrm{d} T^{2}}{T^{4}}+\frac{1}{T^{2}} h, \quad \text { where } \quad h=\frac{\mathrm{d} T^{2}}{1-T^{2}}+\left(1-T^{2}\right) \gamma
$$

The connection $\bar{\nabla}$ given by (2.1) with $\Upsilon=T^{-1} \mathrm{~d} T$ extends to the boundary, and is the Levi-Civita connection of the metric

$$
\begin{aligned}
\bar{g} & =\frac{\mathrm{d} r^{2}}{\left(1+r^{2}\right)^{2}}+\frac{r^{2}}{1+r^{2}} \gamma \\
& =\frac{\mathrm{d} T^{2}}{1-T^{2}}+\left(1-T^{2}\right) \gamma
\end{aligned}
$$

in agreement with Proposition 2.3. The metric $\bar{g}$ extends to the boundary. 
This construction leads to global examples of metric projective compactifications. It follows from the general theory [3] that metrics cones over Einstein manifolds with non-zero Ricci scalar which admit Killing spinors are manifolds with special holonomy. In fact the first examples of non-compact manifolds with exceptional holonomies $G_{2}$ and $\operatorname{Spin}(7)$ were constructed by Bryant [4] as metric cones over the nearly Kähler manifold $S U(3) / T^{2}$ and the weak $G_{2}$ holonomy manifold $S O(5) / S O(3)$, respectively. In view of Theorem 2.4 , these metric cones admit metric projective compactifications.

\section{Para-c-Projective Compactifications of Einstein Metrics}

In [7], the concept of $c$-projective compactification was defined. It is based on almost $c$-projective geometry [10], an analogue of projective geometry defined for almost complex manifolds $(M, J)$ in which the equivalence class of connections defining the $c$-projective structure must be complex and minimal. ${ }^{3}$ Here we review the definition of $c$-projective compactification, modifying to the "para" case where the para-almost-complex structure squares to $I d$ rather than $-I d$. We then introduce a class of $2 n$-dimensional, neutral signature Einstein metrics arising from projective structures in dimension $n$ and show that these admit a compactification which we call para-c-projective.

Although $c$-projective compactification is defined for any almost complex manifold, the definition can be applied to pseudo-Riemannian metrics $g$ which are Hermitian with respect to the almost complex structure so long as there exists a connection which preserves both $g$ and $J$ and has minimal torsion. Such Hermitian metrics are said to be admissible. Note that such a connection, if it exists, is uniquely defined, since the conditions that it be complex and minimal determine its torsion. It is thus given by the Levi-Civita connection of $g$ plus a constant multiple of the Nijenhuis tensor of $J$.

We make the following definition in the para- $c$-projective case.

Definition 3.1. Let $(M, g, J)$ be a para-Hermitian manifold, and let $\nabla^{L}$ be a connection which preserves both $g$ and $J$ and has minimal torsion. The structure $(M, g, J)$ admits a para-c-projective compactification to a manifold with boundary $\bar{M}=M \cup \partial M$, if there exists a function $T: \bar{M} \rightarrow \mathbb{R}$ such that $\mathcal{Z}(T)$ (the set where $T=0$ on $\bar{M}$ ) is the boundary $\partial M \subset \bar{M}$, the differential $\mathrm{d} T$ does not vanish on $\partial M$, and the connection

$$
\begin{gathered}
\bar{\nabla}_{X} Y=\nabla^{L}{ }_{X} Y+\Upsilon(X) Y+\Upsilon(J X) J X+\Upsilon(Y) X+\Upsilon(J Y) J X, \text { where } \\
\Upsilon=\frac{\mathrm{d} T}{2 T},
\end{gathered}
$$

extends to $\bar{M}$.

\footnotetext{
${ }^{3}$ Recall that a connection on an almost complex manifold $(M, J)$ is called complex if it preserves $J$ and minimal if the torsion is just the Nijenhuis tensor of $J$ up to a constant factor.
} 
Note that the para-c-projective change of connection $\nabla^{L} \rightarrow \overline{\nabla^{L}}$ differs from the $c$-projective case in the signs of some of the terms, to account for the fact that $J$ squares to the $I d$ rather than $-I d$.

It follows easily from this definition that the endomorphism $J$ on $M$ naturally extends to all of $\bar{M}$ by parallel transport with respect to $\overline{\nabla^{L}}$. It thus defines an almost para-CR structure on the hypersurface distribution $\mathcal{D}$ defined by $\mathcal{D}_{x}:=T_{x} \partial M \cap J\left(T_{x} \partial M\right)$. It can be shown (see Lemma 5 of [7] and modify to the case $J^{2}=I d$ ) that this almost para-CR structure is non-degenerate if and only if for any local defining function $T$ the one-form $\theta=\mathrm{d} T \circ J$ restricts to a contact form on $\partial M$.

The first main result of [7] is Theorem 8 in this reference, which gives a local form for an admissible Hermitian metric which is sufficient for the corresponding $c$-projective structure to be $c$-projectively compact. The theorem is stated below, adapted to the para- $c$-projective case. Note that this includes an assumption that the Nijenhuis tensor $\mathcal{N}$ of $J$ takes so-called asymptotically tangential values. This is equivalent to the following statement in index notation:

$$
\left.\left(\mathcal{N}^{a}{ }_{b c} \nabla_{a} T\right)\right|_{T=0}=0 .
$$

The following result arises by a trivial adaption of the arguments in [7] for the almost complex case, and so further details may be obtained from that source.

Theorem 3.2 [7]. Let $\bar{M}$ be a smooth manifold with boundary $\partial M$ and interior $M$. Let $J$ be an almost para-complex structure on $\bar{M}$, such that $\partial M$ is non-degenerate and the Nijenhuis tensor $\mathcal{N}$ of $J$ has asymptotically tangential values. Let $g$ be an admissible pseudo-Riemannian Hermitian metric on $M$. For a local defining function $T$ for the boundary defined on an open subset $\mathcal{U} \subset \bar{M}$, put $\theta=\mathrm{d} T \circ J$ and, given a non-zero real constant $C$, define a Hermitian $\left(\begin{array}{l}0 \\ 2\end{array}\right)$-tensor field $h_{T, C}$ on $\mathcal{U} \cap M$ by

$$
h_{T, C}:=T g+\frac{C}{T}\left(\mathrm{~d} T^{2}-\theta^{2}\right) .
$$

Suppose that for each $x \in \partial M$ there is an open neighbourhood $\mathcal{U}$ of $x$ in $\bar{M}, a$ local defining function $T$ defined on $\mathcal{U}$, and a non-zero constant $C$ such that

- $h_{T, C}$ admits a smooth extension to all of $\mathcal{U}$

- for all vector fields $X, Y$ on $U$ with $\mathrm{d} T(Y)=\theta(Y)=0$, the function $h_{T, C}(X, J Y)$ approaches $C \mathrm{~d} \theta(X, Y)$ at the boundary.

Then $g$ is c-projectively compact.

The statement in Theorem 3.2 does not depend on the choice of $T$. Different choices of $T$ result in rescalings of the contact form $\theta$ on the boundary by a nowhere vanishing function.

\section{Example: A Class of Neutral Signature Einstein Metrics}

In this section, we shall introduce a class of para-Hermitian metrics, which we will show to be para- $c$-projectively compact by virtue of Theorem 3.2. 
Definition 4.1. A projective structure $[\nabla]$ on an $n$-dimensional manifold $N$ is an equivalence class of torsion-free affine connection on $N$ which share the same unparametrised geodesics. Two connections $\bar{\nabla}$ and $\nabla$ belong to the same projective class if they are related by (2.1) for some one-form $\Upsilon$ on $N$.

Given a projective structure $[\nabla]$ on an $n$-dimensional manifold $N$, there exists a canonical neutral signature Einstein metric $g$ with non-zero scalar curvature, as well as a symplectic form $\Omega$, on the total space $M$ of a certain rank $n$ affine bundle over $N$. The general construction of this metric and the proof of the projective invariance has been presented in [13] in dimension four, and in [12] in general dimension. Here we shall give an explicit coordinate description from [13]. In Theorem 4.2, we shall show how to recover the metric and the symplectic form (4.1) from the natural pairing on the co-tractor bundle.

The sections of the affine bundle $M \rightarrow N$ are in one-to-one correspondence with the $[\nabla]$ representative connections (see $\S 2.4$ and $\S 3.4$ in [13]), and hence the choice of a representative connection $\nabla \in[\nabla]$ provides a diffeomorphism $T^{*} N \rightarrow M$. Pulling back the pair $(g, \Omega)$ with this diffeomorphism gives a pair $\left(g_{\nabla}, \Omega_{\nabla}\right)$ on $T^{*} N$ which - in local coordinates $\left(x^{i}, \xi_{i}\right)$ on the cotangent bundle $\nu: T^{*} N \rightarrow N$-takes the form

$$
\begin{aligned}
g & =\left(d \xi_{i}-\left(\Gamma_{i j}^{k} \xi_{k}-\xi_{i} \xi_{j}-\mathrm{P}_{(i j)}\right) d x^{i}\right) \odot d x^{j} \\
\Omega & =d \xi_{i} \wedge d x^{i}+\mathrm{P}_{[i j]} d x^{i} \wedge d x^{j}, \quad \text { where } i, j=1, \ldots, n .
\end{aligned}
$$

Here $\Gamma_{j k}^{i}$ denote the Christoffel symbols and $\mathrm{P}_{i j}$ is the Schouten tensor of $\nabla$.

In [13] it was shown that the manifold $M$ can be identified with the complement of an $\mathbb{R} \mathbb{P}^{n-1}$ sub-bundle in the projectivisation $\mathbb{P}\left(\mathcal{T}^{*}\right)$ of a certain rank $(n+1)$ vector bundle (the so-called co-tractor bundle) $\mathcal{T}^{*}$ over $N$. In the special case where $N=\mathbb{R} \mathbb{P}^{n}$, and $[\nabla]$ is projectively flat the manifold $M=$ $S L(n+1, \mathbb{R}) / G L(n, \mathbb{R})$ can be identified with the projection of $\mathbb{R}^{n+1} \times \mathbb{R}_{n+1} \backslash \mathcal{Z}$, where $\mathcal{Z}$ denotes the set of incident pairs (point, hyperplane). See [20] for other applications of (4.1).

The compactification procedure described in Theorem 4.3 will, for the model, attach these incident pairs back to $M$, and more generally (in case of a curved projective structure on $N$ ) will attach the $\mathbb{R P}^{n-1}$ sub-bundle of $\mathbb{P}\left(\mathcal{T}^{*}\right)$. The boundary $\partial M$ from Definition 3.1 will play a role of a submanifold manifold separating two open sets in $\mathbb{P}\left(\mathcal{T}^{*}\right)$ in the sense described in the next subsection.

\subsection{The Constructions in Tractor Terms}

Let the projective structure $(N,[\nabla])$, be represented by some torsion-free affine connection $\nabla$ on $N$, where the latter has dimension at least 2 . Let $\mathcal{E}(1) \rightarrow N$ be the line bundle which is the standard $-2(n+1)$ th root of the square of the canonical bundle of $N$ (which, note, is canonically oriented). For any vector bundle $\mathcal{B}$ and line bundle $\mathcal{E}(w)$ we write $\mathcal{B}(w)$ as a shorthand for $\mathcal{B} \otimes \mathcal{E}(w)$.

Canonically on the projective manifold $(N,[\nabla])$, there is the rank $(n+1)$ co-tractor bundle [2]

$$
\mathcal{T}^{*} \rightarrow N
$$


This has a composition sequence

$$
0 \rightarrow T^{*} N(1) \rightarrow \mathcal{T}^{*} \stackrel{X}{\rightarrow} \mathcal{E}(1) \rightarrow 0
$$

where the map $X \in \Gamma(\mathcal{T}(1))$ is called the (projective) canonical tractor. Choosing a connection in $[\nabla]$ determines a splitting of this sequence and so then have $\mathcal{T}^{*}=\mathcal{E}(1) \oplus T^{*} N(1)$, and we can represent an element $V$ of $\mathcal{T}^{*}$ as a pair $(\sigma, \mu)=[V]_{\nabla}$ (see e.g. [6]). Any other connection $\bar{\nabla}$ in $[\nabla]$ is related to $\nabla$ by (2.1), for some 1-form field $\Upsilon$ on $N$, and the corresponding transformation

$$
[V]_{\nabla}=(\sigma, \mu) \mapsto(\sigma, \mu+\sigma \Upsilon)=[V]_{\bar{\nabla}}
$$

The main importance of $\mathcal{T}^{*}$ is that it admits a canonical projectively invariant tractor connection $\nabla^{\mathcal{T}^{*}}$ given by

$$
\nabla^{\mathcal{T}}{ }_{i}\left(\begin{array}{c}
\sigma \\
\mu_{j}
\end{array}\right)=\left(\begin{array}{c}
\nabla_{i} \sigma-\mu_{i} \\
\nabla_{i} \mu_{j}+\mathrm{P}_{i j} \sigma
\end{array}\right) .
$$

We shall now present two variants of the construction of [13], and then its compactification (to be made precise in Theorem 4.3). We begin with compactification of the construction in [13]. For simplicity, let us assume that $N$ is orientable.

4.1.1. Compactification by Line Projectiviation. On the total space of $\mathcal{T}^{*}$, we pullback $\pi: \mathcal{T}^{*} \rightarrow N$ along $\pi$ to get $\pi^{*}\left(\mathcal{T}^{*}\right) \rightarrow \mathcal{T}^{*}$ as a vector bundle over the total space $\mathcal{T}^{*}$. By construction, this bundle has a tautological section $U \in \Gamma\left(\pi^{*}\left(\mathcal{T}^{*}\right)\right)$. We also have $\pi^{*}(\mathcal{T}(w))$ for any weight $w$, and we shall write simply $X \in \Gamma\left(\pi^{*}(\mathcal{T}(1))\right)$ for the pullback to $\mathcal{T}^{*}$ of the canonical tractor $X$ on $N$.

There is a canonical density $\tau \in \Gamma\left(\pi^{*} \mathcal{E}(1)\right)$ given by

$$
\tau:=X\lrcorner U \text {. }
$$

Now define

$$
\kappa: \mathcal{T}^{*} \longrightarrow \mathcal{M}:=\mathbb{P}\left(\mathcal{T}^{*}\right)
$$

by the fibrewise projectivisation, and use also $\pi$ for the map to $N$ :

$$
\pi: \mathcal{M} \rightarrow N \text {. }
$$

Note that $\tau$ is homogeneous of degree 1 up the fibres of the map $\mathcal{T}^{*} \rightarrow \mathcal{M}$. Thus $\tau$ determines, and is equivalent to, a section (that we also denote) $\tau$ of a certain density bundle $\pi^{*}(\mathcal{E}(1)) \otimes \mathcal{E}_{\mathcal{T}^{*}}(1)$, on $\mathcal{M}$ that for simplicity we shall denote $\mathcal{E}(1,1)$. So $\mathcal{M}$ is stratified according to whether or not $\tau$ is vanishing, and we write $\mathcal{Z}(\tau)$ to denote, in particular, the zero locus of $\tau$.

To elaborate on the densities used here, and their generalisation to arbitrary weights: By $\mathcal{E}_{\mathcal{T}^{*}}\left(w^{\prime}\right)$, for $w^{\prime} \in \mathbb{R}$, we mean the line bundle on $\mathbb{P}\left(\mathcal{T}^{*}\right)$ whose sections correspond to functions $f: \pi^{*} \mathcal{T}^{*} \rightarrow \mathbb{R}$ that are homogeneous of degree $w$ in the fibres of $\pi^{*} \mathcal{T}^{*} \rightarrow \mathbb{P}\left(\mathcal{T}^{*}\right)$. Then for any weight $w$ we also have $\mathcal{E}(w)$ on $N$ and its pull back to the bundle $\pi^{*} \mathcal{E}(w) \rightarrow \mathbb{P}\left(\mathcal{T}^{*}\right)$. Then

$$
\mathcal{E}\left(w, w^{\prime}\right):=\pi^{*} \mathcal{E}(w) \otimes \mathcal{E}_{\mathcal{T} *}\left(w^{\prime}\right) .
$$

Using these tools, we can recover the metric of [13]: 
Theorem 4.2. There is a neutral signature metric on $\mathcal{M} \backslash \mathcal{Z}(\tau)$ determined by the canonical pairing of the horizontal and vertical subspaces of $T\left(\mathcal{T}^{*}\right)$. This metric is Einstein, with non-zero Ricci scalar, and agrees with (4.1).

Proof. Considering first the total space $\mathcal{T}^{*}$ and then its tangent bundle, note that there is an exact sequence

$$
0 \rightarrow \pi^{*} \mathcal{T}^{*} \rightarrow T\left(\mathcal{T}^{*}\right) \rightarrow \pi^{*} T N \rightarrow 0,
$$

where we have identified $\pi^{*} \mathcal{T}^{*}$ as the vertical sub-bundle of $T\left(\mathcal{T}^{*}\right)$. A connection on the vector bundle $\mathcal{T}^{*} \rightarrow N$ is equivalent to a splitting of this sequence; a connection identifies $\pi^{*} T N$ with a distinguished sub-bundle of horizontal subspaces in $T\left(\mathcal{T}^{*}\right)$. Thus, in particular, the projective tractor connection on $\mathcal{T}^{*} \rightarrow N$ gives a canonical splitting of the sequence (4.6). So we have

$$
T\left(\mathcal{T}^{*}\right)=\pi^{*} T N \oplus \pi^{*} \mathcal{T}^{*} .
$$

We move now to the total space of $\mathbb{P} \mathcal{T}^{*}$, and we note that again the tractor (equivalently, Cartan) connection determines a splitting of the tangent bundle $T\left(\mathbb{P} \mathcal{T}^{*}\right)$, see [8]. From the usual Euler sequence of projective space (or see (4.26) in the last section), it follows that for $T\left(\mathbb{P} \mathcal{T}^{*}\right)$ the second term of the display (4.7) is replaced by a quotient of $\pi^{*} \mathcal{T}^{*}(0,1)$. Indeed, if we work at a point $p \in \mathbb{P}\left(\mathcal{T}^{*}\right)$, observe that $\pi^{*} \mathcal{T}^{*}(0,1)$ has a filtration

$$
\left.\left.0 \rightarrow \mathcal{E}(0,0)_{p} \stackrel{U_{p}}{\rightarrow} \pi^{*} \mathcal{T}^{*}(0,1)\right|_{p} \rightarrow \pi^{*} \mathcal{T}^{*}(0,1)\right|_{p} /\left\langle U_{p}\right\rangle \rightarrow 0
$$

where, as usual, $U$ is the canonical section. But away from $\mathcal{Z}(\tau)$, we have that $U$ canonically splits the appropriately re-weighted pull back of the sequence $(4.2)$

$$
0 \rightarrow \pi^{*} T^{*} N(1,1) \rightarrow \pi^{*} \mathcal{T}^{*}(0,1) \stackrel{X / \tau}{\rightarrow} \mathcal{E}(0,0) \rightarrow 0
$$

This identifies the quotient in (4.8), and thus we have canonically

$$
T\left(\mathbb{P}\left(\mathcal{T}^{*}\right) \backslash \mathcal{Z}(\tau)\right)=\pi^{*} T N \oplus \pi^{*} T^{*} N(1,1) .
$$

It follows that on $\mathcal{M}$ there is canonically a metric $\boldsymbol{g}$ and symplectic form $\boldsymbol{\Omega}$ taking values in $\mathcal{E}(1,1)$, given by

$$
\begin{aligned}
\boldsymbol{g}\left(w_{1}, w_{2}\right) & \left.\left.=\frac{1}{2}\left(\Pi_{H}\left(w_{1}\right)\right\lrcorner \Pi_{V}\left(w_{2}\right)+\Pi_{H}\left(w_{2}\right)\right\lrcorner \Pi_{V}\left(w_{1}\right)\right) \quad \text { and } \\
\boldsymbol{\Omega}\left(w_{1}, w_{2}\right) & \left.\left.=\frac{1}{2}\left(\Pi_{H}\left(w_{1}\right)\right\lrcorner \Pi_{V}\left(w_{2}\right)-\Pi_{H}\left(w_{2}\right)\right\lrcorner \Pi_{V}\left(w_{1}\right)\right)
\end{aligned}
$$

where

$$
\Pi_{H}: T(\mathcal{M} \backslash \mathcal{Z}(\tau)) \rightarrow \pi^{*} T N \quad \text { and } \quad \Pi_{V}: T(\mathcal{M} \backslash \mathcal{Z}(\tau)) \rightarrow \pi^{*} T^{*} N(1,1)
$$

are the projections. Then we obtain the metric and symplectic form by

$$
g:=\frac{1}{\tau} \boldsymbol{g} \quad \text { and } \quad \Omega:=\frac{1}{\tau} \boldsymbol{\Omega} .
$$

What remains to be done is to show that (4.9) agrees with the normal form (4.1) once a trivialisation of $\mathcal{T}^{*} \rightarrow N$ has been chosen.

Let $p \in N$ and let $\mathcal{W} \subset N$ be an open neighbourhood of $p$ with local coordinates $\left(x^{1}, \ldots, x^{n}\right)$ such that $T_{p} N=\operatorname{span}\left(\partial / \partial x^{1}, \ldots, \partial / \partial x^{n}\right)$. The 
connection (4.4) gives a splitting of $T\left(\mathcal{T}^{*}\right)$ into the horizontal and vertical sub-bundles

$$
T\left(\mathcal{T}^{*}\right)=H\left(\mathcal{T}^{*}\right) \oplus V\left(\mathcal{T}^{*}\right)
$$

as in (4.7). To obtain the explicit form of this splitting, let $V_{\alpha}, \alpha=0,1, \ldots, n$ be components of a local section of $\mathcal{T}^{*}$ in the trivialisation over $\mathcal{W}$. Then

$$
\nabla^{\mathcal{T}^{*}} V_{\beta}=d V_{\beta}-\gamma_{\beta}^{\alpha} V_{\alpha},
$$

where $\gamma_{\alpha}^{\beta}=\gamma_{i \alpha}^{\beta} \mathrm{d} x^{i}$, and the components of the co-tractor connection $\gamma_{i \alpha}^{\beta}$ are given in terms of the connection $\nabla$ on $N$, and its Schouten tensor, and can be read-off from (4.4):

$$
\gamma_{i 0}^{0}=0, \quad \gamma_{i 0}^{j}=\delta_{i}^{j}, \quad \gamma_{i j}^{k}=\Gamma_{i j}^{k}, \quad \gamma_{i j}^{0}=-\mathrm{P}_{i j} .
$$

In terms of these components, we can write

$$
\begin{aligned}
H\left(\mathcal{T}^{*}\right) & =\operatorname{span}\left(\frac{\partial}{\partial x^{i}}+\gamma_{i \alpha}^{\beta} V_{\beta} \frac{\partial}{\partial V_{\alpha}}, i=1, \ldots, n\right), \\
V\left(\mathcal{T}^{*}\right) & =\operatorname{span}\left(\frac{\partial}{\partial V_{\alpha}}, \alpha=0,1, \ldots, n\right) .
\end{aligned}
$$

Setting $\xi_{i}=V_{i} / V_{0}$, where $\tau=V_{0} \neq 0$ on the complement of $\mathcal{Z}(\tau)$, we can compute the push forwards of these spaces to $\mathbb{P}\left(\mathcal{T}^{*}\right) \backslash \mathcal{Z}(\tau)$ :

$$
\begin{aligned}
\kappa_{*} H\left(\mathcal{T}^{*}\right) & =\operatorname{span}\left(h_{i} \equiv \frac{\partial}{\partial x^{i}}-\left(\mathrm{P}_{i j}+\xi_{i} \xi_{j}-\Gamma_{i j}^{k} \xi_{k}\right) \frac{\partial}{\partial \xi_{j}}\right) \\
\kappa_{*} V\left(\mathcal{T}^{*}\right) & =\operatorname{span}\left(v^{i} \equiv \frac{\partial}{\partial \xi_{i}}\right) .
\end{aligned}
$$

The non-zero components of the metric (4.9) are given by

$$
g\left(v^{i}, h_{j}\right)=\delta^{i}{ }_{j}
$$

which indeed agrees with (4.1) which is known to be Einstein [13].

Next we observe that $\mathbb{P}\left(\mathcal{T}^{*}\right) \backslash \mathcal{Z}(\tau)$ is an affine bundle modelled on $T^{*} N$. The point is that given $\nabla$ in the projective class there is a smooth fibre bundle isomorphism

$$
\iota: T^{*} N \rightarrow \mathbb{P}\left(\mathcal{T}^{*}\right) \backslash \mathcal{Z}(\tau)
$$

First, given $\nabla$, we can represent an element $U \in \mathcal{T}_{p}^{*}(p \in N)$ by the pair $(\tau, \mu) \in \mathcal{E}(1)_{p} \oplus T_{p}^{*} N(1)$, or, if we choose coordinates on $N$, by collection

$$
U=\left(\tau, \mu_{i}\right), \quad i=1, \ldots, n .
$$

Then, dropping the choice $\nabla \in[\nabla], U \in \mathcal{T}_{p}^{*}$ is an equivalence class of such pairs by the equivalence relation (4.3) that covers the equivalence relation between elements of $[\nabla]$.

Thus, given $\nabla$, and from the naturality of all maps, it follows that the total space of $T^{*} N$ can be identified with $\mathbb{P}\left(\mathcal{T}^{*}\right) \backslash \mathcal{Z}(\tau)$ by (for each $p \in N$ )

$$
T_{p}^{*} N \ni \xi_{i} \mapsto\left[\left(1, \xi_{i}\right)\right]=\left[\left(\tau, \tau \xi_{i}\right)\right] \in \mathbb{P}\left(\mathcal{T}^{*}\right) \backslash \mathcal{Z}(\tau) .
$$

Thus we may view $\mathcal{M}$ as a compactification of $T^{*} N$ and, by construction, this is a closed manifold iff $N$ is closed. 
Note that by this construction, it is easily verified that the zero locus of $\tau$ is a smoothly embedded hypersurface in $\mathcal{M}$, and from (4.2) it follows at once that this may be identified with the total space of the fibrewise projectivisation $\mathbb{P}\left(T^{*} N\right)$ (which is well known to have a para-CR structure).

A feature of this construction is that in each dimension $n$ (of $N$ ) either the hypersurface $\mathcal{Z}(\tau)$ (if $n$ odd) is not orientable, or $\mathcal{M}$ (if $n$ even) is not orientable.

4.1.2. Compactification by Ray-Projectiviation. Instead we may follow the construction above but instead define $\mathcal{M}:=\pi^{*}\left(\mathbb{P}_{+}\left(\mathcal{T}^{*}\right)\right)$, where $\mathbb{P}_{+}\left(\mathcal{T}^{*}\right)$ is the ray-projectivisation of $\mathcal{T}^{*}$ (i.e. the fibres of $\mathcal{T}^{*} \rightarrow \mathbb{P}_{+}\left(\mathcal{T}^{*}\right)$ are isomorphic to $\left.\mathbb{R}_{+}\right)$. Then the bundles $\mathcal{E}\left(w, w^{\prime}\right)$ should also be defined via ray homogeneity. In this case, for $N$ orientable, both $\mathcal{Z}(\tau)$ and $\mathcal{M}$ are orientable, and again $\mathcal{M}$ is closed iff $N$ is. Now from (4.2) we have that $\mathcal{Z}(\tau)$ may be identified with the fibrewise ray-projectivisation $\mathbb{P}_{+}\left(T^{*} N\right)$. In this variant of the construction, there are two copies $M_{ \pm}$of $T^{*} N$ in $\mathcal{M}$ according to the sign of $\tau$. Moreover, each of $\mathcal{M} \backslash M_{\mp}$ is a manifold that is globally a para-c-projective compactification of $M_{ \pm}$in sense of Theorem 3.2.

4.1.3. Remark on Continuing the Tractor Approach. It would be possible to achieve our main aims by continuing the tractor approach. We will not pursue this here as we want to emphasise that with little effort the main result now follows directly form the properties of the metric. However, we sketch just the basic idea: By our construction above, it follows that $\mathcal{M}$ has a canonical para-c-projective geometry. In the notation as above, $\pi^{*} \mathcal{T} \oplus \pi^{*} \mathcal{T}^{*}$ is the corresponding para-c-projective tractor bundle and this has a canonical tractor connection that trivially extends (in fibre directions) the pull back of the projective connection (that is available in horizontal directions). The dual-pairing between $\pi^{*} \mathcal{T}$ and $\pi^{*} \mathcal{T}^{*}$ determines a fibre metric and compatible symplectic form on the bundle $\pi^{*} \mathcal{T} \oplus \pi^{*} \mathcal{T}^{*}$ and this is obviously preserved by the connection. What remains is to show that the tractor connection so constructed satisfies properties that mean that it is normal in the sense defined in e.g. [9]. With this established then the main results then follow from the general holonomy theory in [8].

\subsection{The Main Theorem}

In the previous Sect. 4.1.2, we have presented a candidate $\bar{M} \equiv \mathcal{M} \backslash M_{\mp}$ for a para-c-projective compactification of the Einstein para-Kahler manifold $(M, g, \Omega)$ given by (4.1). What remains to be done is to show that near the boundary $\mathcal{Z}(\tau)=0$ of $\bar{M}$ the metric (4.1) can be put in the local normal form of Theorem 3.2.

The endomorphism $J: T M \rightarrow T M$ defined by $\Omega(X, Y)=g(J X, Y)$ satisfies $J^{2}=I d$, and the associated Libermann connection $\nabla^{L}$ [16] is given by

$$
\nabla^{L}{ }_{a} X_{b}=\nabla^{\mathbf{g}}{ }_{a} X_{b}-G^{c}{ }_{a b} X_{c}, \quad \text { where } \quad G^{c}{ }_{a b}=-\Omega^{c d} \nabla^{\mathbf{g}}{ }_{d} \Omega_{a b}
$$


and $\nabla^{\mathbf{g}}$ is the Levi-Civita connection of $g$. This connection is metric, has minimal torsion, and preserves the almost para-complex structure $J$. It thus belongs to a para-c-projective equivalence class which we will show to be compactifiable in the sense of Theorem 3.2.

Theorem 4.3. The Einstein almost para-Kähler metric $(M, g, \Omega)$ given by (4.1) admits a para-c-projective compactification $\bar{M}$. The structure on the $(2 n-$ 1)-dimensional boundary $\partial M \cong \mathbb{P}\left(T^{*} N\right)$ of $\bar{M}$ includes a contact structure together with a conformal structure and a para-CR structure defined on the contact distribution.

Proof. In the proof below, we shall explicitly construct the boundary $\partial M$ together with the contact structure and the associated conformal structure on the contact distribution. We shall first deal with the model $M=S L(n+1) / G L(n)$ and then explain how the curvature of $(N,[\nabla])$ modifies the compactification.

In the model case, we can define coordinates $x^{i}$ on $N=\mathbb{R} \mathbb{P}^{n}$ by taking $X=\left(1, x^{1}, \ldots, x^{n}\right)$, where $\left(X^{0}, \ldots, X^{n}\right)$ are homogeneous coordinates and we are working in an open set where $X^{0} \neq 0$. The $x^{i}$ are flat coordinates, so the connection components (and hence the Schouten tensor) vanish and (4.1) reduces to

$$
g=d \xi_{i} \odot \mathrm{d} x^{i}+\xi_{i} \xi_{j} \mathrm{~d} x^{i} \odot \mathrm{d} x^{j}, \quad \Omega=d \xi_{i} \wedge d x^{i} \quad \text { where } \quad i, j=1, \ldots, n .
$$

We can relate [13] the affine coordinates $\xi_{i}$ on the fibres of $T^{*} N$ to the tractor coordinates (4.11) by setting $\xi_{i}=\mu_{i} / \tau$ on the complement of the zero locus $\mathcal{Z}(\tau)$ of $\tau$.

Now consider an open set $\mathcal{U} \subset M$ given by $\xi_{i} x^{i}>0$, and define the function $T$ on $\mathcal{U}$ by

$$
T=\frac{1}{\xi_{i} x^{i}} .
$$

We shall attach a boundary $\partial \mathcal{U}$ to the open set $\mathcal{U}$ such that $T$ extends to a function $\bar{T}$ on $\mathcal{U} \cup \partial \mathcal{U}$, and $\bar{T}$ is the defining function for this boundary. We then investigate the geometry on $M$ in the limit $T \rightarrow 0$. It is clear from above that the zero locus of $\bar{T}$ will be contained in the zero locus $\mathcal{Z}(\tau)$ of $\tau$, and therefore belongs to the boundary of $\bar{M}$. We will use $\bar{T}$ as a defining function for $\bar{M}$ in an open set $\overline{\mathcal{U}} \subset \bar{M}$. The strategy of the proof is to extend $T$ to a coordinate system on $\mathcal{U}$, such that near the boundary the metric $g$ takes a form as in Theorem 3.2.

First define $\theta \in \Lambda^{1}(\bar{M})$ by

$$
V\lrcorner \theta=J(V)\lrcorner d T, \quad \text { or equivalently } \quad \theta_{a}=\Omega_{a c} g^{b c} \nabla^{\mathbf{g}} T, \quad a, b, c=1, \ldots, 2 n
$$

where $J$ is the para-complex structure of $(g, \Omega)$. Using $(4.14)$ this gives

$$
\theta=2 T(1-T) \xi_{i} d x^{i}-\mathrm{d} T .
$$

We need $n$ open sets $U_{1}, \ldots, U_{n}$ such that $\xi_{k} \neq 0$ on $U_{k}$ to cover the zero locus of $T$. Here we chose $k=n$, and adapt a coordinate system (which we will prove to be Pfaff) given by

$$
\left(T, Z_{1}, \ldots, Z_{n-1}, X^{1}, \ldots, X^{n-1}, Y\right),
$$


where $T$ is given by (4.15) and

$$
Z_{A}=\frac{\xi_{A}}{\xi_{n}}, \quad X^{A}=x^{A}, \quad Y=x^{n}, \quad \text { where } \quad A=1, \ldots, n-1 .
$$

We compute

$$
\theta=2(1-T) \frac{\mathrm{d} Y+Z_{A} \mathrm{~d} X^{A}}{K}-\mathrm{d} T, \quad \xi_{n}=\frac{1}{K T}, \quad \text { where } \quad K \equiv Y+Z_{A} X^{A},
$$

and substitute

$$
\xi_{i} \mathrm{~d} x^{i}=\frac{1}{K T}\left(\mathrm{~d} Y+Z_{A} \mathrm{~d} X^{A}\right)
$$

into (4.14). This gives

where

$$
g=\frac{\theta^{2}-\mathrm{d} T^{2}}{4 T^{2}}+\frac{1}{T} h
$$

$h=\frac{1}{4(1-T)}\left(\theta^{2}-\mathrm{d} T^{2}\right)+\frac{1}{K}\left(\mathrm{~d} Z_{A} \odot \mathrm{d} X^{A}-\frac{1}{2(1-T)} X^{A} \mathrm{~d} Z_{A} \odot(\theta+\mathrm{d} T)\right)$

is regular at the boundary $T=0$. This is in agreement with the asymptotic form in Theorem 3.2 (see [7] for further details).

The restriction $h$ to $\partial M$ gives a metric on a distribution $\mathcal{D}=\operatorname{Ker}\left(\left.\theta\right|_{T=0}\right)$

$$
\begin{aligned}
\left.\theta\right|_{T=0}= & 2 \frac{\mathrm{d} Y+Z_{A} \mathrm{~d} X^{A}}{Y+Z_{A} X^{A}}, \quad h_{0}=\frac{1}{4}\left(\left.\theta\right|_{T=0}\right)^{2}+\frac{1}{2\left(Y+Z_{A} X^{A}\right)} \\
& \left(2 \mathrm{~d} Z_{A} \odot \mathrm{d} X^{A}-X^{A} \mathrm{~d} Z_{A} \odot\left(\left.\theta\right|_{T=0}\right)\right) .
\end{aligned}
$$

Note that $T$ is only defined up to multiplication by a positive function. Changing the defining function in this way results in a conformal rescalling of $\left.\theta\right|_{T=0}$, thus the metric on the contact distribution is also defined up to an overall conformal scale. We shall choose the scale so that the contact form is given by $\left.\theta_{0} \equiv K \theta\right|_{T=0}$ on $T(\partial M)$, with the metric on $\mathcal{D}$ given by

$$
h_{\mathcal{D}}=\mathrm{d} Z_{A} \odot \mathrm{d} X^{A} .
$$

We now move on to deal with the curved case where the metric on $M$ is given by (4.1). The coordinate system $\left(T, Z_{A}, X^{A}, Y\right)$ is as above, and the one-form $\theta$ in (4.16) is given by

$$
\theta=2 T(1-T) \xi_{i} \mathrm{~d} x^{i}-\mathrm{d} T+2 T^{2}\left(\mathrm{P}_{i j}-\Gamma_{i j}^{k} \xi_{k}\right) x^{i} \mathrm{~d} x^{j},
$$

or in the $\left(T, Z_{A}, X^{A}, Y\right)$ coordinates,

$$
\begin{aligned}
\theta= & 2(1-T) \frac{Z_{A} \mathrm{~d} X^{A}+\mathrm{d} Y}{K}-\mathrm{d} T \\
& +2 T^{2}\left[\left(\mathrm{P}_{A B}-\frac{\Gamma_{A B}^{C} Z_{C}+\Gamma_{A B}^{n}}{T K}\right) X^{A} \mathrm{~d} X^{B}+\left(\mathrm{P}_{n B}-\frac{\Gamma_{n B}^{C} Z_{C}+\Gamma_{n B}^{n}}{T K}\right) Y \mathrm{~d} X^{B}\right. \\
& \left.+\left(\mathrm{P}_{A n}-\frac{\Gamma_{A n}^{C} Z_{C}+\Gamma_{A n}^{n}}{T K}\right) X^{A} \mathrm{~d} Y+\left(\mathrm{P}_{n n}-\frac{\Gamma_{n n}^{C} Z_{C}+\Gamma_{n n}^{n}}{T K}\right) Y \mathrm{~d} Y\right]
\end{aligned}
$$


Guided by the formula (4.17), we define

$$
h=T g-\frac{1}{4 T}\left(\theta^{2}-\mathrm{d} T^{2}\right),
$$

which we find to be

$$
\begin{aligned}
h=\frac{1}{4(1-T)}\left(\theta^{2}-\mathrm{d} T^{2}\right)+\frac{1}{K}\left(\mathrm{~d} Z_{A} \odot \mathrm{d} X^{A}-\frac{1}{2(1-T)} X^{A} \mathrm{~d} Z_{A} \odot(\theta+\mathrm{d} T)\right) \\
\quad-\frac{1}{K}\left(\left(\Gamma_{A B}^{C} Z_{C}+\Gamma_{A B}^{n}\right) \mathrm{d} X^{A} \odot \mathrm{d} X^{B}+\left(\Gamma_{n n}^{C} Z_{C}+\Gamma_{n n}^{n}\right) \mathrm{d} Y \odot \mathrm{d} Y\right. \\
\left.+2\left(\Gamma_{A n}^{C} Z_{C}+\Gamma_{A n}^{n}\right) \mathrm{d} X^{A} \odot \mathrm{d} Y\right) \\
+T\left(\mathrm{P}_{A B} \mathrm{~d} X^{A} \odot \mathrm{d} X^{B}+2 \mathrm{P}_{A n} \mathrm{~d} X^{A} \odot \mathrm{d} Y+\mathrm{P}_{n n} \mathrm{~d} Y \odot \mathrm{d} Y\right) .
\end{aligned}
$$

This is smooth as $T \rightarrow 0$.

Restricting $h$ to $T=0$ yields a metric which differs from (4.18) by the curved contribution given by the components of the connection, but not the Schouten tensor. Substituting $\mathrm{d} Y=\left.K \theta\right|_{T=0} / 2-Z_{A} \mathrm{~d} X^{A}$, disregarding the terms involving $\left.\theta\right|_{T=0}$ in $h$, and conformally rescalling by $K$ yields the metric

$$
\begin{aligned}
h_{\mathcal{D}} & =\left(\mathrm{d} Z_{A}-\Theta_{A B} \mathrm{~d} X^{B}\right) \odot \mathrm{d} X^{A}, \quad \text { where } \\
\Theta_{A B} & =\Gamma_{A B}^{C} Z_{C}+\Gamma_{A B}^{n}+\left(\Gamma_{n n}^{C} Z_{C}+\Gamma_{n n}^{n}\right) Z_{A} Z_{B}-2\left(\Gamma_{A n}^{C} Z_{C}+\Gamma_{A n}^{n}\right) Z_{B}
\end{aligned}
$$

defined on the contact distribution $\mathcal{D}=\operatorname{Ker}\left(\theta_{0}\right)$, where $\theta_{0}=2\left(\mathrm{~d} Y+Z_{A} \mathrm{~d} X^{A}\right)$.

We now invoke Theorem 3.2, verifying by explicit computation that the remaining two conditions are satisfied. The first of these conditions is that the metric $h_{\mathcal{D}}$ is compatible with the Levi-form of the almost para-CR structure induced on $\partial M$ by $J$, i.e.

$$
h_{\mathcal{D}}(X, Y)=\mathrm{d} \theta_{0}(J X, Y), \quad \text { for } \quad X \in \mathcal{D} .
$$

The second is that the Nijenhuis tensor takes asymptotically tangential values, i.e. that (3.1) is satisfied.

Both of these follow from computing the para-complex structure $J$ in the $\left(T, Z_{A}, X^{A}, Y\right)$ coordinates. We find

$$
\begin{aligned}
\left.J\right|_{T=0}= & -\frac{\partial}{\partial X^{A}} \otimes \mathrm{d} X^{A}+\frac{\partial}{\partial Y} \otimes \mathrm{d} Y+\frac{\partial}{\partial Z_{A}} \otimes \mathrm{d} Z_{A}+\frac{\partial}{\partial T} \otimes \mathrm{d} T \\
& -\frac{Z_{B}}{K} \frac{\partial}{\partial T} \otimes \mathrm{d} X^{B}-\frac{1}{K} \frac{\partial}{\partial T} \otimes \mathrm{d} Y \\
& -\left(\Gamma_{A B}^{D} Z_{D}+\Gamma_{A B}^{n}\right) \frac{\partial}{\partial Z_{A}} \otimes \mathrm{d} X^{B}+\left(\Gamma_{n B}^{D} Z_{D}+\Gamma_{n B}^{n}\right) Z_{C} \frac{\partial}{\partial Z_{C}} \otimes \mathrm{d} X^{B} \\
& -\left(\Gamma_{A n}^{D} Z_{D}+\Gamma_{A n}^{n}\right) \frac{\partial}{\partial Z_{A}} \otimes \mathrm{d} Y+\left(\Gamma_{n n}^{D} Z_{D}+\Gamma_{n n}^{n}\right) Z_{C} \frac{\partial}{\partial Z_{C}} \otimes \mathrm{d} Y .
\end{aligned}
$$


Restricting to vectors in $\mathcal{D}$ amounts to substituting $\mathrm{d} Y=\theta_{0} / 2-Z_{A} \mathrm{~d} X^{A}$ and disregarding the terms involving $\theta_{0}$ as above, so that

$$
\begin{aligned}
\left.J\right|_{\mathcal{D}}= & -\frac{\partial}{\partial X^{A}} \otimes \mathrm{d} X^{A}+Z_{A} \frac{\partial}{\partial Y} \otimes \mathrm{d} X^{A}+\frac{\partial}{\partial Z_{A}} \otimes \mathrm{d} Z_{A}+\frac{\partial}{\partial T} \otimes \mathrm{d} T \\
& -\frac{2 Z_{B}}{K} \frac{\partial}{\partial T} \otimes \mathrm{d} X^{B}-\Theta_{A B} \frac{\partial}{\partial Z_{A}} \otimes \mathrm{d} X^{B}
\end{aligned}
$$

and (4.22) is satisfied.

For the Nijenhuis condition, we use the formula

$$
\mathcal{N}_{b c}^{a}=J_{[b}^{d} \partial_{|d|} J_{c]}^{a}-J_{[b}^{d} \partial_{c]} J_{d}^{a} .
$$

Note that we need only consider components of this with $a=T$, and thus only need to work with the $\partial / \partial T$ components of $J$ to find the terms which look like $\partial J$. This is a one-form which we shall call $J^{(T)}$ and find to be

$$
\begin{aligned}
J^{(T)}= & \left(-\frac{Z_{B}}{K}+\frac{T\left[2 Z_{B}+\left(\Gamma_{A B}^{D} Z_{D}+\Gamma_{A B}^{n}\right) X^{A}+\left(\Gamma_{n B}^{D} Z_{D}+\Gamma_{n B}^{n}\right) Y\right]}{K}\right. \\
& \left.-T^{2}\left[\mathrm{P}_{A B} X^{A}+\mathrm{P}_{n B} Y\right]\right) \mathrm{d} X^{B} \\
& \left(-\frac{1}{K}+\frac{T\left[2+\left(\Gamma_{A n}^{D} Z_{D}+\Gamma_{A n}^{n}\right) X^{A}+\left(\Gamma_{n n}^{D} Z_{D}+\Gamma_{n n}^{n}\right) Y\right]}{K}\right. \\
& \left.-T^{2}\left[\mathrm{P}_{A n} X^{A}+\mathrm{P}_{n n} Y\right]\right) \mathrm{d} Y .
\end{aligned}
$$

Note that this agrees with (4.23) when $T=0$. We use it to calculate $\mathcal{N}^{a}{ }_{b c} \nabla_{a} T$, dropping terms which vanish when $T=0$ to verify (3.1).

\subsection{Two-Dimensional Projective Structures}

In the case if $n=2$ the coordinates on $\partial M$ are $(X, Y, Z)$, and (4.21) yields $h_{\mathcal{D}}=\mathrm{d} Z \odot \mathrm{d} X-\left[\Gamma_{11}^{2}+\left(\Gamma_{11}^{1}-2 \Gamma_{12}^{2}\right) Z+\left(\Gamma_{22}^{2}-2 \Gamma_{12}^{2}\right) Z^{2}+\Gamma_{22}^{1} Z^{3}\right] \mathrm{d} X \odot \mathrm{d} X$,

which is transparently invariant under the projective changes

$$
\Gamma_{i j}^{k} \longrightarrow \Gamma_{i j}^{k}+\delta_{i}^{k} \Upsilon_{j}+\delta_{j}^{k} \Upsilon_{i}
$$

of $\nabla$. In the two-dimensional case, the projective structures $(N,[\nabla])$ are equivalent to second-order ODEs which are cubic in the first derivatives (see, e.g. [5])

$$
\frac{d^{2} Y}{d X^{2}}=\Gamma_{22}^{1}\left(\frac{d Y}{d X}\right)^{3}+\left(2 \Gamma_{12}^{1}-\Gamma_{22}^{2}\right)\left(\frac{d Y}{d X}\right)^{2}+\left(\Gamma_{11}^{1}-2 \Gamma_{12}^{2}\right)\left(\frac{d Y}{d X}\right)-\Gamma_{11}^{2},
$$

where the integral curves of (4.24) are the unparametrised geodesics of $\nabla$. The integral curves $C$ of (4.24) are integral submanifolds of a differential ideal $\mathcal{I}=<\theta_{0}, \theta_{1}>$, where

$$
\begin{aligned}
\theta_{0}= & \mathrm{d} Y+Z \mathrm{~d} X, \theta_{1}=\mathrm{d} Z \\
& -\left(\Gamma_{11}^{2}+\left(\Gamma_{11}^{1}-2 \Gamma_{12}^{2}\right) Z+\left(\Gamma_{22}^{2}-2 \Gamma_{12}^{1}\right) Z^{2}+\Gamma_{22}^{1} Z^{3}\right) \mathrm{d} X
\end{aligned}
$$

are one-forms on a three-dimensional manifold $B=\mathbb{P}\left(T^{*} N\right)$ with local coordinates $(X, Y, Z)$. If $f: C \rightarrow B$ is an immersion, then $f^{*}\left(\theta_{0}\right)=0, f^{*}\left(\theta_{1}\right)=0$ is 
equivalent to (4.24) as long as $\theta_{2} \equiv \mathrm{d} X$ does not vanish. In terms of these three one-forms the contact structure, and the metric on the contact distribution are given by $\theta_{0}, h_{\mathcal{D}}=\theta_{1} \odot \theta_{2}$.

\subsection{The Model via an Orbit Decomposition}

In this section, we describe here the flat (in the sense of parabolic geometries) model $[11,13]$ of our construction in tractor terms.

The flat projective structure on $N=\mathbb{R P}^{n}$ gives rise to the neutral signature para-Kähler Einstein metric on $M=S L(n+1) / G L(n)$

$$
g=d \xi_{i} \odot \mathrm{d} x^{i}+\left(\xi_{i} \mathrm{~d} x^{i}\right)^{2}, \quad \Omega=d \xi_{i} \wedge \mathrm{d} x^{i}, \quad \text { where } \quad i, j, \ldots=1, \ldots, n .
$$

In [13], §7.1 it was explained how this homogeneous model corresponds to the projectivised co-tractor bundle of $\mathbb{R P}^{n}$, with an $\mathbb{R P}_{n-1}$ removed from each $\mathbb{R P}_{n}$ fibre. This $\mathbb{R P}_{n-1}$ corresponds to incident pairs of points and hyperplanes in $\mathbb{R}^{n+1} \times \mathbb{R}_{n+1}$.

Here we shall instead take $N$ to be the sphere $S^{n}$ with its standard projective structure as this is orientable in all dimensions and, more importantly, on this (double cover of $\mathbb{R P}^{n}$ ) the tractor bundle is trivial, and this simplifies the discussion. The underlying space of the (compactified) model of dimension $2 n$ is $S^{n} \times S_{n}$ where both $S_{n}$ and $S^{n}$ denote spheres that are dual as we shall explain.

Consider first two vector spaces each isomorphic to $\mathbb{R}^{n+1}$ :

$$
V \cong \mathbb{R}^{n+1} \quad \mathrm{~W} \cong \mathbb{R}^{n+1}
$$

and view each as a representation space for an $S L(n+1, \mathbb{R})$ action. So $G:=$ $S L(V) \times S L(W)$ acts on $V \times W$. (Note that we may wlog consider $V$ and $W$ as, respectively, the \pm 1 eigenspaces of the single vector space $\mathbb{V}:=V \oplus W$ equipped with a $\mathbb{J}$ s.t. $\mathbb{J}^{2}=1$.)

Now the action of $S L(V)$ descends to a transitive action on the ray projectivisation $\mathbb{P}_{+}(V)$ and similarly $S L(W)$ acts transitively on $\mathbb{P}_{+}(W)$. Thus $G:=S L(V) \times S L(W)$ acts transitively on the manifold

$$
\mathcal{M}:=\mathbb{P}_{+}(V) \times \mathbb{P}_{+}(W) .
$$

We can represent an element of $\mathcal{M}$ in terms of pairs of homogeneous coordinates $([Y],[Z])$ where $0 \neq Y \in V$ and $0 \neq Z \in W$.

Note that as a smooth manifold $\mathcal{M}=S^{n} \times S^{n}$, but as a homogeneous manifold it is

$$
G / P=\left(S L(V) / P_{X}\right) \times\left(S L(W) / P_{U}\right)
$$

where $P_{X}$ (resp. $P_{U}$ ) is the parabolic subgroup in $S L(V)$ that stabilises a point $[X]$ in $\mathbb{P}_{+}(V)$ (resp. $[U] \in \mathbb{P}_{+}(W)$ ), and $P$ is the group product $P_{X} \times P_{W}$ which itself is a parabolic subgroup of the semisimple group $G$.

Now introduce an additional structure which breaks the $G$ symmetry. Namely we fix an isomorphism

$$
I: W \rightarrow V^{*}
$$


where $V^{*}$ denotes the dual space to $V$. The subgroup $H \cong S L(n+1, \mathbb{R})$ of $G$ that fixes this may be identified with $S L(V)$ which acts on a pair $(Y, Z) \in$ $V \times V^{*}$ by the defining representation and on the first factor and by the dual representation on the second factor.

Given this structure we may now (suppress $I$ and) view $\mathcal{M}$ as consisting of pairs $([X],[U])$ where $0 \neq X \in V$ and $0 \neq U \in V^{*}$. That is

$$
\mathcal{M}=\mathbb{P}_{+}(V) \times \mathbb{P}_{+}\left(V^{*}\right) .
$$

This is useful as follows: Each element $[U]$ in $\mathbb{P}_{+}\left(V^{*}\right)$ determines an oriented hyperplane in $V$ and each $[X] \in \mathbb{P}_{+}(V)$ an oriented line in $V$. So now we consider the $H$ action on $M$. This has two open orbits and a closed orbit. The last is the incidence space

$$
\mathcal{Z}=\{([X],[U]) \in \mathcal{M} \mid U(X)=0\}
$$

which sits as smooth orientable separating hypersurface in $\mathcal{M}$. Then there are the open orbits

$$
\begin{aligned}
& M_{+}=\{([X],[U]) \in \mathcal{M} \mid U(X)>0\} \quad \text { and } \\
& M_{-}=\{([X],[U]) \in \mathcal{M} \mid U(X)<0\} .
\end{aligned}
$$

We may think of $\mathcal{Z}$ as the 'boundary' (at infinity) for the open orbits $M_{ \pm}$.

We now describe the geometries on the orbits. The claim is that there are Einstein metrics in $M_{ \pm}$, while $\mathcal{Z}$ is well known as the model for so-called contact Langrangian (or sometimes called para-CR) geometry, this is a real analogue of hypersurface-type CR geometry.

First observe that $N_{V}:=\mathbb{P}_{+}(V)$ is the flat model of projective geometry. So in particular we have

$$
0 \rightarrow \mathcal{E}_{V}(-1) \stackrel{X}{\rightarrow} \mathcal{T}_{V} \rightarrow T N_{V}(-1) \rightarrow 0
$$

where $\mathcal{T}_{V}$ is the projective tractor bundle on $N_{V}$ and $X$ is the tautological section of $\mathcal{T}(1)$, which coincides with the canonical tractor. Similarly there is a sequence on $N^{W}:=\mathbb{P}_{+}\left(V^{*}\right)$

$$
0 \rightarrow \mathcal{E}^{W}(-1) \stackrel{U}{\rightarrow} \mathcal{T}^{W} \rightarrow T N^{W}(-1) \rightarrow 0 .
$$

There is a natural tractor bundle $\mathcal{T}:=\mathcal{T}_{V} \oplus \mathcal{T}^{W}$ on $M$, where $X$ and $U$ are not incident this induces a metric on $M$ as follows. Observe that, at a point $([X],[U])$ where $X\lrcorner U \neq 0$, the tractor field $U$ splits the first sequence by $\nu \in \Gamma(\mathcal{E}(-1,0))$ defined by

$$
\nu:=U / \tau
$$

with $\tau:=X\lrcorner U$ (and where we have used an obvious weight notation). This follows as $X\lrcorner \nu=1$. Similarly

$$
x:=X / \tau \in \Gamma(\mathcal{E}(0,-1))
$$

splits the second short exact sequence because $x\lrcorner U=1$. Thus we obtain a neutral signature metric on $T N_{V} \oplus T N^{W}$ by these two steps: First, using these splittings yields a bundle monomorphism

$$
T N_{V}(-1,0) \oplus T N^{W}(0,-1) \rightarrow \mathcal{T}_{V} \oplus \mathcal{T}^{W} .
$$


Second, this gives a symmetric form $\boldsymbol{g}$ and symplectic form $\boldsymbol{\Omega}$ on $T N_{V}(-1,0) \oplus$ $T N^{W}(0,-1)$ by then using the canonical metric and symplectic form on $\mathcal{T}_{V} \oplus$ $\mathcal{T}^{W}$ given by the duality of $\mathcal{T}_{V}$ and $\mathcal{T}^{W}$. Thus $\boldsymbol{g} \in \Gamma\left(S^{2} T^{*} M(1,1)\right)$ and $\boldsymbol{\Omega} \in$ $\Gamma\left(\Lambda^{2} T^{*} M(1,1)\right)$. Then set

$$
g:=\frac{1}{\tau} \boldsymbol{g} \quad \text { and } \quad \Omega:=\frac{1}{\tau} \boldsymbol{\Omega} .
$$

The metric $g$ is easily seen to have neutral signature. It is Einstein because the tractor metric on $\mathcal{T}$ is parallel for the tractor connection (see [8] for the analogous c-projective case). The tractor connection arises from the usual parallel transport on the vector space $V \oplus V^{*}$ viewed as an affine manifold.

\section{Acknowledgements}

The work of MD has been partially supported by STFC consolidated grant no. ST/P000681/1. ARG gratefully acknowledges support from the Royal Society of New Zealand via Marsden Grants 13-UOA-113 and 16-UOA-051. AW is grateful for support from the Sims Fund. MD acknowledges the hospitality of the University of Auckland where this work has started, and ARG acknowledges the hospitality of the University of Cambridge where it has finished. MD is grateful to Dmitri Alekseevsky and Vladimir Matveev for useful correspondence.

Open Access. This article is licensed under a Creative Commons Attribution 4.0 International License, which permits use, sharing, adaptation, distribution and reproduction in any medium or format, as long as you give appropriate credit to the original author(s) and the source, provide a link to the Creative Commons licence, and indicate if changes were made. The images or other third party material in this article are included in the article's Creative Commons licence, unless indicated otherwise in a credit line to the material. If material is not included in the article's Creative Commons licence and your intended use is not permitted by statutory regulation or exceeds the permitted use, you will need to obtain permission directly from the copyright holder. To view a copy of this licence, visit http://creativecommons. org/licenses/by/4.0/.

Publisher's Note Springer Nature remains neutral with regard to jurisdictional claims in published maps and institutional affiliations.

\section{References}

[1] Atiyah, M., Dunajski, M., Mason, L.: Twistor theory at fifty: from contour integrals to twistor strings. In: Proceedings of the Royal Society, vol. 473 (2017). arXiv:1704.07464

[2] Bailey, T.N., Eastwood, M.G., Gover, A.R.: Thomas's structure bundle for conformal, projective and related structures. Rocky Mt. J. Math. 24, 1-27 (1994)

[3] Bar, C.: Real killing spinors and holonomy. Commun. Math. Phys. 154, 509-521 (1993) 
[4] Bryant, R.L.: Metrics with exceptional holonomy. Ann. Math. 126, 525-576 (1987)

[5] Bryant, R.L., Dunajski, M., Eastwood, M.G.: Metrisability of two-dimensional projective structures. J. Differ. Geom. 83, 465-499 (2009)

[6] Čap, A., Gover, A.R.: Projective compactifications and Einstein metrics. J. Reine Angew. Math. 717, 47-75 (2016)

[7] Čap, A., Gover, A.R.: C-projective compactification; (quasi-)Kaehler metrics and CR boundaries. Am J Math (2016) arXiv:1603.07039

[8] Čap, A., Gover, A.R., Hammerl, M.: Holonomy reductions of Cartan geometries and curved orbit decompositions. Duke Math J 163(5), 1035-1070 (2014). arXiv:1103.4497

[9] Čap, A., Slovák, J.: Parabolic geometries I: background and general theory 2009. American Mathematical Society, Providence (2009)

[10] Calderbank, D.M.J., Eastwood, M.G., Matveev, V.S., Neusser, K.: C-projective geometry (2016). arXiv:1512.04516

[11] Casey, S., Dunajski, M., Tod, K.P.: Twistor geometry of a pair of second order ODEs Comm. Math. Phys. 321, 681-701 (2013)

[12] Dunajski, M., Waterhouse, A.: Einstein metrics, projective structures and the $S U(\infty)$ Toda equation. J. Geom. Phys. 147, 103523 (2020). arXiv:1906.11570

[13] Dunajski, M., Mettler, T.: Gauge theory on projective surfaces and antiself-dual Einstein metrics in dimension four. J. Geom. Anal. 1-32 (2017). arXiv:1509.04276

[14] Eguchi, T., Hanson, A.J.: Asymptotically flat self-dual solutions to Euclidean gravity. Phys. Lett. B 74, 249-251 (1978)

[15] Gray, A., Hervella, L.M.: The sixteen classes of almost Hermitian manifolds and their linear invariants. Ann. Mat. Pura Appl. 123, 35-58 (1980)

[16] Libermann, P.: Sur le probleme d'equivalence de certaines structures infinitesimales. Ann. Mat. Pura Appl. 36, 27-120 (1954)

[17] Levi-Civita, T.: On the transformations of the dynamical equations. Ann. Math. 24, 255-300 (1896)

[18] Matveev, V.S., Rosemann, S.: The degree of mobility of Einstein metrics (2015). arXiv:1503.00968

[19] Melrose, R.B.: Geometric Scattering Theory. Stanford Lectures. Cambridge University Press, Cambridge (1995)

[20] Mettler, T.: Extremal conformal structures on projective surfaces (2015). arXiv:1510.01043

[21] Penrose, R.: Zero rest-mass fields including gravitation: asymptotic behaviour. Proc. R. Soc. Lond. Ser. A284, 159-203 (1965)

[22] Uhlenbeck, K.: Removable singularities in Yang-Mills fields. Commun. Math. Phys. 83, 11-29 (1982)

[23] Witten, E.: Anti de Sitter space and holography. Adv. Theor. Math. Phys. 2, 253-291 (1998) 
Maciej Dunajski and Alice Waterhouse

Department of Applied Mathematics and Theoretical Physics

University of Cambridge

Wilberforce Road

Cambridge CB3 0WA

UK

e-mail: m.dunajski@damtp.cam.ac.uk;

Alice Waterhouse

e-mail: aw592@cam.ac.uk

A. Rod Gover

Department of Mathematics

The University of Auckland

Private Bag 92019

Auckland 1142

New Zealand

e-mail: r.gover@auckland.ac.nz

Communicated by Mihalis Dafermos.

Received: September 12, 2018.

Accepted: February 10, 2020. 